03

\title{
Ударные волны в термоупругой среде с точечными дефектами
}

\author{
() В.И. Ерофреев,,$^{1,2}$ A.В. Леонтьева, ${ }^{2}$ А.В. Шекоян ${ }^{3}$ \\ ${ }^{1}$ Научно-исследовательский институт механики Национального исследовательского Нижегородского \\ государственного университета им. Н.И. Лобачевского, \\ 603950 Нижний Новгород, Россия \\ ${ }^{2}$ Институт проблем машиностроения РАН - филиал Федерального исследовательского центра \\ „Институт прикладной фризики РАН“, \\ 603024 Нижний Новгород, Россия \\ ${ }^{3}$ Институт механики НАН Республики Армения, \\ 0019 Ереван, Армения \\ e-mail: erof.vi@yandex.ru
}

Поступило в Редакцию 15 мая 2018 г.

В окончательной редакции 12 апреля 2019 г.

Принято к публикации 14 мая 2019 г.

Изучено распространение плоских продольных волн в безграничной среде с точечными дефектами, находящейся в нестационарном неоднородном температурном поле. Рассмотрена самосогласованная задача, учитывающая как влияние акустической волны на образование и перемещение дефектов, так и влияние дефектов на особенности распространения акустической волны. Показано, что в случае отсутствия диффузии тепла система уравнений сводится к нелинейному эволюционному уравнению относительно смещений частиц среды. Уравнение можно считать формальным обобщением уравнения Кортевега-де Вриза-Бюргерса. Методом усеченных разложений найдено точное решение эволюционного уравнения в виде стационарной ударной волны с монотонным убыванием. Отмечено, что диссипативные эффекты, обусловленные наличием дефектов, преобладают над дисперсией, связанной с миграцией дефектов в среде.

Ключевые слова: термоупругая волна, стационарная ударная волна, точечные дефекты, эволюционное уравнение.

DOI: $10.21883 /$ JTF.2020.01.48656.190-19

\section{Введение}

При воздействии на материал лазерного излучения или потока частиц (например, при ионной имплантации) в нем создаются точечные дефекты (вакансии, межузлия) [1]. Прохождение интенсивной продольной акустической волны способствует изменению в областях растяжения и сжатия энергии активации образования точечных дефектов, приводя к их пространственному перераспределению. Дефекты, мигрирующие по материалу, рекомбинируют на различного рода центрах. Роль таких центров могут играть дислокации, примеси внедрения и др.

Волновые эффекты в ансамблях дислокаций изучались в работах [2-9]. Также в [6] рассматривались задачи распространения упругой волны в вязкотермоупругом композите с шариковыми неоднородностями.

В [10] показано, что задачу о распространении акустической волны в материале с точечными дефектами следует рассматривать как самосогласованную, включающую в себя, наряду с динамическим уравнением теории упругости, кинетическое уравнение для плотности дефектов.

В [11] исследовано взаимодействие нелинейной волны деформации с полем концентрации точечных дефектов (вакансий, межузлий), приводящее как к рассеянию вол- ны, так и к изменению энергии активации образования дефектов и их пространственному перераспределению.

При этом предполагалось, что основными процессами, определяющими поведение дефектов, являются процессы генерации, рекомбинации и диффузии. Объемная взаимная рекомбинация разноименных дефектов не учитывалась.

Распространение нелинейных продольных волн в пластине при учете взаимодействия продольной компоненты смещений среды с полями температуры и концентрации неравновесных атомных точечных дефектов исследованы в [12].

В [13] исследовано воздействие дислокаций и точечных дефектов на пространственную локализацию нелинейных акустических волн, распространяющихся в материалах. Влияние дефектов на пространственную локализацию нелинейных волн с учетом взаимной рекомбинации разноименных дефектов изучалось в [14]. В работах показано, что вакансии и межузлия способствуют формированию пространственно локализованных нелинейных волн.

В настоящей работе мы рассматриваем распространение плоской продольной волны в безграничной среде с точечными дефектами, при образовании которых меняется температура среды. Объемную взаимную рекомбинацию разноименных дефектов не учитываем. Считаем, 
что в среде возникают дефекты только одного типа либо вакансии, либо межузлия.

\section{Постановка задачи}

Для получения самосогласованной динамической задачи для термоупругой среды с точечными дефектами к уравнениям теории упругости

$$
\rho \frac{\partial^{2} u_{i}}{\partial t^{2}}=\frac{\partial \sigma_{i j}}{\partial x_{j}}
$$

и теплопроводности

$$
T \frac{\partial S}{\partial t}=\kappa \Delta \Theta
$$

где $u_{i}$ - компоненты вектора перемещений, $\sigma_{i j}-$ компоненты тензора напряжений, $\Theta=T-T_{0}, T_{0}-$ начальная температура среды, $T$ - текущая температура, $\kappa-$ коэффициент теплопроводности, $\rho-$ плотность материала, $S$ - энтропия единицы объема среды, следует добавить кинетические уравнения, описывающие изменение числа точечных дефектов в единице объема [1]:

$$
\begin{aligned}
\frac{\partial n_{1}}{\partial t}= & q_{01}+q_{1}\left(\frac{\partial u_{1}}{\partial x_{1}}+\frac{\partial u_{2}}{\partial x_{2}}+\frac{\partial u_{3}}{\partial x_{3}}\right)+D_{1} \Delta n_{1} \\
& -\beta_{1} n_{1}-\beta_{12} n_{2} \\
\frac{\partial n_{2}}{\partial t}= & q_{02}+q_{2}\left(\frac{\partial u_{1}}{\partial x_{1}}+\frac{\partial u_{2}}{\partial x_{2}}+\frac{\partial u_{3}}{\partial x_{3}}\right)+D_{2} \Delta n_{2} \\
& -\beta_{2} n_{2}-\beta_{21} n_{1} .
\end{aligned}
$$

Здесь $n_{1}, n_{2}$ - объемные концентрации вакансий и межузельных атомов в единице объема среды соответственно, $q_{01}, q_{02}$ - темпы генерации точечных дефектов при отсутствии деформации, $D_{1}, D_{2}-$ коэффициенты диффузии вакансий и межузельных атомов соответственно, $\beta_{12}, \beta_{21}$ - скорости взаимной рекомбинации дефектов типа „межузельные атомы-вакансия“ и „вакансия-межузельные атомы“ соответственно, $\beta_{1}, \beta_{2}$ - скорости рекомбинации дефектов на стоках, $q_{1}, q_{2}$ - коэффициенты взаимодействия деформации с дефектами.

Свободная энергия единицы объема среды имеет следующий вид:

$$
\begin{gathered}
F=F_{0}+\frac{\lambda}{2} u_{l l}^{2}+\mu\left(u_{i k}-\frac{1}{2} \delta_{i k} u_{l l}\right)^{2}-d_{1} u_{l l} n_{1}-d_{2} u_{l l} n_{2} \\
-\gamma \Theta u_{l l}+m_{1} n_{1} \Theta+m_{2} n_{2} \Theta-\frac{c_{p}}{T_{0}^{2}} \Theta^{2}+\frac{A}{2} u_{i k} u_{i l} u_{k l} \\
+B u_{i k}^{2} u_{l l}+\frac{C}{3} u_{l l}^{3}+\frac{q_{3}}{2} u_{l l}^{2} n_{1}+q_{4} u_{l l} n_{1}^{2} \\
+\frac{q_{5}}{2} u_{l l}^{2} n_{2}+q_{6} u_{l l} n_{2}^{2} .
\end{gathered}
$$

где $F_{0}-$ свободная энергия единицы объема среды до возмущения, $\lambda$ и $\mu-$ константы Ламе, $d_{1}, d_{2}-$ коэффициенты, характеризующие взаимодействие упругой волны с дефектами, $\gamma$ - термический коэффициент, $m_{1}, m_{2}$ - коэффициенты, характеризующие взаимодействие ,дефект-изменение температуры“, $c_{p}$ удельная теплоемкость материала, $A, B, C$ - константы Ландау третьего порядка, $q_{3}, q_{4}, q_{5}$ (как и ранее введенные $\left.q_{1}, q_{2}\right)$ - коэффициенты, характеризующие взаимодействие деформации с дефектами.

Компоненты тензора деформаций $u_{i k}$ и перемещения $u_{i}$ связаны соотношениями, учитывающими геометрическую нелинейность [15]:

$$
u_{i k}=\frac{1}{2}\left(\frac{\partial u_{i}}{\partial x_{k}}+\frac{\partial u_{k}}{\partial x_{i}}+\frac{\partial u_{l}}{\partial x_{i}} \frac{\partial u_{l}}{\partial x_{k}}\right) .
$$

Входящие в (1), (2) напряжения и энтропия определяются из соотношения (5):

$$
\begin{gathered}
\sigma_{i k}=-\frac{\partial F}{\partial\left(\frac{\partial u_{i}}{\partial x_{k}}\right)}, \\
S=\frac{\partial F}{\partial T} .
\end{gathered}
$$

При описании распространения вдоль координаты $x_{3}$ плоской волны система (1)-(8) преобразуется к виду

$$
\begin{gathered}
\rho \frac{\partial^{2} u_{3}}{\partial t^{2}}-a \frac{\partial^{2} u_{3}}{\partial x_{3}^{2}}-\beta_{N} \frac{\partial u_{3}}{\partial x_{3}} \frac{\partial^{2} u_{3}}{\partial x_{3}^{2}}=-\gamma \frac{\partial \Theta}{\partial x_{3}}-d_{j} \frac{\partial n_{j}}{\partial x_{3}} \\
c_{\varepsilon} \frac{\partial \theta}{\partial t}-\chi \frac{\partial^{2} \theta}{\partial x_{3}^{2}}=-\gamma T_{0} \frac{\partial^{2} u_{3}}{\partial x_{3} \partial t}-T_{0} p_{j} \frac{\partial n_{j}}{\partial t} \\
\frac{\partial n_{j}}{\partial t}=q_{\varepsilon} \frac{\partial u_{3}}{\partial x_{3}}+D_{j} \frac{\partial^{2} n_{j}}{\partial x_{3}^{2}}-\beta_{j} n_{j}
\end{gathered}
$$

где $u_{3}\left(x_{3}, t\right)$ - смещение частиц среды вдоль координаты $x_{3}, \theta\left(x_{3}, t\right)$ - перепад температуры $\left(\theta=T-T_{0}\right)$, $T_{0}$ - температура естественного состояния, $n_{j}\left(x_{3}, t\right)-$ объемная концентрация точечных дефектов $j$-го типа $(j=V$ - для вакансий, $j=i-$ для межузлий $)$, $\rho$ - плотность, $a=\lambda+2 \mu, \lambda, \mu-$ постоянные Ламе, $c_{l}=\sqrt{a / \rho}-$ скорость распространения продольной волны в отсутствие дефектов, $\beta_{N}-$ коэффициент нелинейности $\left(\beta_{N}=3 \lambda+6 \mu+2 A+6 B+2 C\right), A, B, C-$ модули Ландау третьего порядка, $\gamma$ - термический коэффициент, $d_{j}=(\lambda+(2 / 3) \mu) \Omega_{j}, \Omega_{j}$ - дилатационный параметр, характеризующий изменение объема среды при образовании в нем одного точечного дефекта $\left(\Omega_{V}<0\right.$, $\left.\Omega_{i}>0\right), c_{\varepsilon}-$ теплопроводность при постоянной деформации, $\chi-$ коэффициент теплопроводности, $p_{j}-$ теплоемкость дефекта типа $j, q_{\varepsilon}$ - темп генерации точечных дефектов при наличии деформации, $D_{j}-$ коэффициент диффузии дефекта типа $j, \beta_{j}-$ скорость рекомбинации на стоках дефекта $j$-го типа.

Системой уравнений (9)-(11) описывается распространение плоской продольной волны в среде как с 
дефектами типа „вакансия“, так и с дефектами типа „межузлие“. Системы отличаются между собой знаками перед последним слагаемым первого уравнения системы. При дальнейшем анализе рассматриваются оба случая.

Пусть коэффициент теплопроводности является малой величиной $(\chi=0)$, тогда из второго уравнения системы получаем выражение для температуры

$$
\theta\left(x_{3}, t\right)=-\frac{T_{0}}{c_{\varepsilon}}\left(\gamma \frac{\partial u_{3}}{\partial x_{3}}+p_{j} n_{j}\right) .
$$

С учетом (12) систему (9), (11) сводим к одному уравнению относительно смещений частиц среды

$$
\begin{gathered}
\frac{\partial^{2} u_{3}}{\partial t^{2}}-\left(\left(\frac{a}{\rho}+\frac{\gamma^{2} T_{0}}{\rho c_{\varepsilon}}\right)+\frac{q_{\varepsilon}}{\rho \beta_{j}}\left(\frac{\gamma T_{0} p_{j}}{c_{\varepsilon}}-d_{j}\right)\right) \frac{\partial^{2} u_{3}}{\partial x_{3}^{2}} \\
+\frac{1}{\beta_{j}}\left(\frac{\partial}{\partial t}-D_{j} \frac{\partial^{2}}{\partial x_{3}^{2}}\right)\left(\frac{\partial^{2} u_{3}}{\partial t^{2}}-\left(\frac{a}{\rho}+\frac{\gamma^{2} T_{0}}{\rho c_{\varepsilon}}\right) \frac{\partial^{2} u_{3}}{\partial x_{3}^{2}}\right) \\
-\frac{\beta_{N}}{\rho}\left(\frac{\partial u_{3}}{\partial x_{3}} \frac{\partial^{2} u_{3}}{\partial x_{3}^{2}}+\frac{1}{\beta_{j}} \frac{\partial}{\partial x_{3}}\left(\frac{\partial u_{3}}{\partial x_{3}} \frac{\partial^{2} u_{3}}{\partial x_{3} \partial t}\right)\right) \\
+\frac{\beta_{N} D_{j}}{\rho \beta_{j}} \frac{\partial^{2}}{\partial x_{3}^{2}}\left(\frac{\partial u_{3}}{\partial x_{3}} \frac{\partial^{2} u_{3}}{\partial x_{3}^{2}}\right)=0 .
\end{gathered}
$$

В уравнении (13) введем безразмерные величины для продольного перемещения, координаты и времени соответственно $U=u_{3} / u_{0}, z=x_{3} / X, \tau=t / T$. Уравнение примет вид

$$
\begin{aligned}
& \frac{\partial^{2} U}{\partial \tau^{2}}-\frac{\partial^{2} U}{\partial z^{2}}+\frac{\partial}{\partial \tau}\left(\frac{\partial^{2} U}{\partial \tau^{2}}-a_{1} \frac{\partial^{2} U}{\partial z^{2}}\right)-a_{2} \frac{\partial^{2}}{\partial z^{2}} \\
& \times\left(\frac{\partial^{2} U}{\partial \tau^{2}}-a_{1} \frac{\partial^{2} U}{\partial z^{2}}\right)-a_{3}\left(\frac{\partial U}{\partial z} \frac{\partial^{2} U}{\partial z^{2}}+\frac{\partial}{\partial z}\left(\frac{\partial U}{\partial z} \frac{\partial^{2} U}{\partial z \partial \tau}\right)\right) \\
& +a_{2} a_{3} \frac{\partial^{2}}{\partial z^{2}}\left(\frac{\partial U}{\partial z} \frac{\partial^{2} U}{\partial z^{2}}\right)=0
\end{aligned}
$$

где $a_{i}$ - безразмерные комплексы параметров, имеющие вид

$$
\begin{gathered}
a_{1}=\left(\frac{a}{\rho}+\frac{\gamma^{2} T_{0}}{\rho c_{\varepsilon}}\right)\left(\frac{a}{\rho}+\frac{\gamma^{2} T_{0}}{\rho c_{\varepsilon}}+\frac{q_{\varepsilon}}{\rho \beta_{j}}\left(\frac{\gamma T_{0} p_{j}}{c_{\varepsilon}}-d_{j}\right)\right)^{-1}, \\
a_{2}=D_{j} \beta_{j}\left(\frac{a}{\rho}+\frac{\gamma^{2} T_{0}}{\rho c_{\varepsilon}}+\frac{q_{\varepsilon}}{\rho \beta_{j}}\left(\frac{\gamma T_{0} p_{j}}{c_{\varepsilon}}-d_{j}\right)\right)^{-1}, \\
a_{3}=\frac{\beta_{j} \beta_{N} u_{0}}{\rho}\left(\frac{a}{\rho}+\frac{\gamma^{2} T_{0}}{\rho c_{\varepsilon}}+\frac{q_{\varepsilon}}{\rho \beta_{j}}\left(\frac{\gamma T_{0} p_{j}}{c_{\varepsilon}}-d_{j}\right)\right)^{-3 / 2} .
\end{gathered}
$$

В качестве характерных величин длины и времени приняты соответственно

$$
X=\frac{1}{\beta_{j}} \sqrt{\frac{a}{\rho}+\frac{\gamma^{2} T_{0}}{\rho c_{\varepsilon}}+\frac{q_{\varepsilon}}{\rho \beta_{j}}\left(\frac{\gamma T_{0} p_{j}}{c_{\varepsilon}}-d_{j}\right)}, \quad T=\frac{1}{\beta} .
$$

В уравнении (14) диссипативные слагаемые (производные третьего порядка) обусловлены наличием дефектов в среде, дисперсионные (производные четвертого порядка) - диффузионными процессами, протекающими в среде. Полагаем, что диффузионные процессы протекают медленнее, чем процессы взаимодействия дефектов с акустической волной.

\section{Эволюционное уравнение}

Перейдем в уравнении (14) в движущуюся систему координат $\xi=z-c \tau, \eta=\varepsilon \tau$, где $c-$ скорость волны, заранее не известная, $\varepsilon-$ малый параметр $(\varepsilon \ll 1)$. Выбор переменных объясняется тем, что возмущение, распространяясь со скоростью $c$ вдоль оси $z$, медленно эволюционирует во времени из-за нелинейности, дисперсии и диссипации. Считая, что в уравнении (14) все нелинейные и диссипативные слагаемые - малые величины порядка $\varepsilon$, получаем в первом приближении по $\varepsilon$ эволюционное уравнение относительно функции продольной деформации $W=\partial U / \partial \xi$ :

$$
\begin{aligned}
\frac{\partial W}{\partial \eta} & +\frac{\left(1-a_{1}\right)}{2 \varepsilon} \frac{\partial^{2} W}{\partial \xi^{2}}+\frac{a_{2}\left(1-a_{1}\right)}{2 \varepsilon} \frac{\partial^{3} W}{\partial \xi^{3}} \\
& +\frac{a_{3}}{2 \varepsilon}\left(W \frac{\partial W}{\partial \xi}-\frac{\partial}{\partial \xi}\left(W \frac{\partial W}{\partial \xi}\right)\right)=0 .
\end{aligned}
$$

Из нулевого приближении найдена скорость волны $c=1$ (измеряется в относительных единицах), которая учтена в (15). Полученное уравнение можно классифицировать как уравнение Кортевега-де Вриза-Бюргерса (далее - „КдВ-Бюргерса“) с дополнительным нелинейным слагаемым. Поскольку в уравнении (15) содержатся диссипативные и нелинейные слагаемые, возможно, оно имеет решение в виде ударных волн.

\section{Стационарная ударная волна}

Среди возможных решений уравнения (15) существенный интерес представляют решения из класса стационарных волн. Такие волны распространяются с постоянной скоростью и не меняют своей формы в процессе распространения. Уравнение стационарных волн имеет вид

$$
\begin{aligned}
\frac{\partial W}{\partial \chi} & -\frac{\left(1-a_{1}\right)}{2 \varepsilon v} \frac{d^{2} W}{d \chi^{2}}-\frac{a_{2}\left(1-a_{1}\right)}{2 \varepsilon v} \frac{d^{3} W}{d \chi^{3}} \\
& -\frac{a_{3}}{2 \varepsilon v}\left(W \frac{\partial W}{\partial \chi}-\frac{d}{d \chi}\left(W \frac{d W}{d \chi}\right)\right)=0
\end{aligned}
$$


или после интегрирования

$$
\begin{gathered}
W-\frac{\left(1-a_{1}\right)}{2 \varepsilon v} \frac{d W}{d \chi}-\frac{a_{2}\left(1-a_{1}\right)}{2 \varepsilon v} \frac{d^{2} W}{d \chi^{2}} \\
-\frac{a_{3}}{2 \varepsilon v}\left(\frac{W^{2}}{2}-W \frac{d W}{d \chi}\right)=0 .
\end{gathered}
$$

где $W=W(\chi), \chi-$ бегущая координата, $\chi=\xi-v \eta$, $v$ - скорость стационарной волны, бегущей в положительном направлении координатной оси. Константу интегрирования положили равной нулю.

Решение уравнения (17) ищем методом усеченных разложений, подробно описанным в $[16,17]$. Метод используется для поиска точных решений нелинейных дифференциальных уравнений.

Поскольку порядок полюса общего решения равен единице, решение уравнения (17) можно искать в виде

$$
W(\chi)=b_{0} Y(\chi)+b_{1},
$$

где $Y(\chi)=\sqrt{B_{0}} \operatorname{th}\left(\sqrt{b_{0}} \chi\right)$ - решение уравнения Риккати $Y^{\prime}(\chi)=-Y^{2}(\chi)+B_{0}$. Подставляя решение (18) в уравнение (17) и учитывая уравнение Риккати, получаем полином третьей степени относительно $Y(\chi)$. Приравнивая коэффициенты полинома нулю, находим неизвестные коэффициенты

$$
\begin{gathered}
b_{0}=-\frac{2 a_{2}}{a_{3}}\left(1-a_{1}\right), \quad b_{1}=\frac{1}{a_{3}}\left(1-a_{1}\right)\left(1+a_{2}\right), \\
B_{0}=\frac{1}{4 a_{2}^{2}}\left(1+a_{2}\right)^{2} .
\end{gathered}
$$

Одновременно с коэффициентами $b_{0}, b_{1}, B_{0}$ находим выражение для скорости стационарной волны

$$
v=\frac{1}{2 \varepsilon}\left(1-a_{1}\right)\left(1+a_{2}\right) .
$$

Только при этом значении скорости удается найти решение уравнения (17) аналитическим методом. Решение (18) принимает вид

$$
W(\chi)=\frac{1}{a_{3}}\left(1-a_{1}\right)\left(1+a_{2}\right)\left(1-\operatorname{th}\left(\frac{1+a_{2}}{2 a_{2}} \chi\right)\right) .
$$

График зависимости (20) представлен на рис. 1. Анализ функций первой и второй производных $\left(W^{\prime}(\chi)\right.$ и $\left.W^{\prime \prime}(\chi)\right)$ показывает их четность и нечетность соответственно, что говорит о симметрии графиков функций относительно оси ординат и начала координат. График зависимости $W(\chi)$ имеет вид монотонного симметричного относительно точки перегиба кинка. Точка симметрии кинка лежит на оси ординат. Амплитуда $A$ и ширина $\Delta$ кинка равны

$$
A=\frac{2}{a_{3}}\left(1-a_{1}\right)\left(1+a_{2}\right), \quad \Delta=\frac{2 a_{2}}{1+a_{2}} .
$$

Фазовый портрет уравнения (17) представлен на рис. 2. Решение уравнения (20) на рисунке отмечено

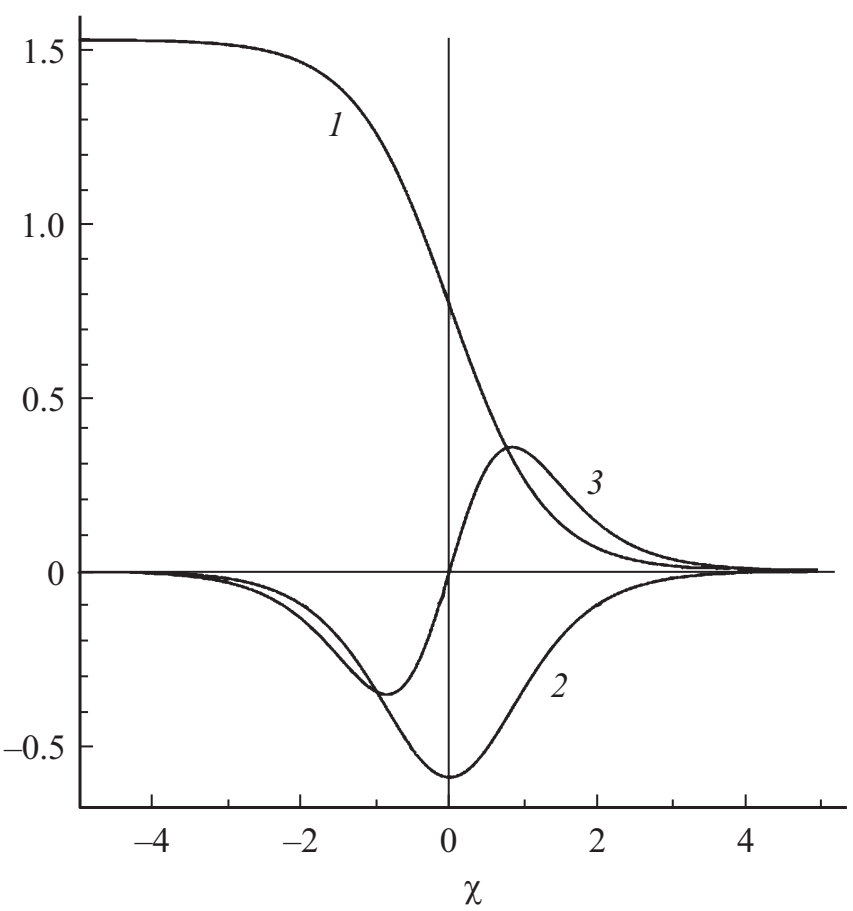

Рис. 1. Зависимости $W(\chi)(1), W^{\prime}(\chi)(2), W^{\prime \prime}(\chi)(3)$ при фиксированных значениях параметров.

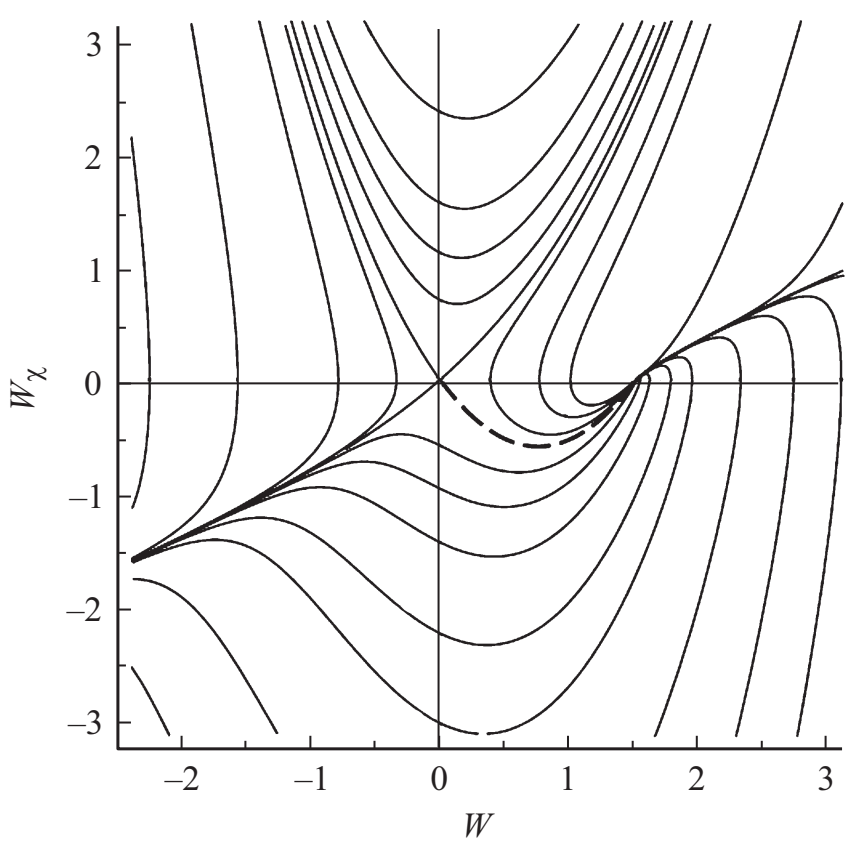

Рис. 2. Фазовый портрет в плоскости $\left(W, W^{\prime}\right)$.

штрихом. Из рисунка видно, что на фазовом портрете есть два состояния равновесия. Исследование на устойчивость состояний равновесия показывает, что одно из состояний равновесия „узел“, другое - „седло“. Движение происходит из неустойчивого состояния равновесия „узел“ (при $\chi \rightarrow-\infty)$ в седловую точку (при 


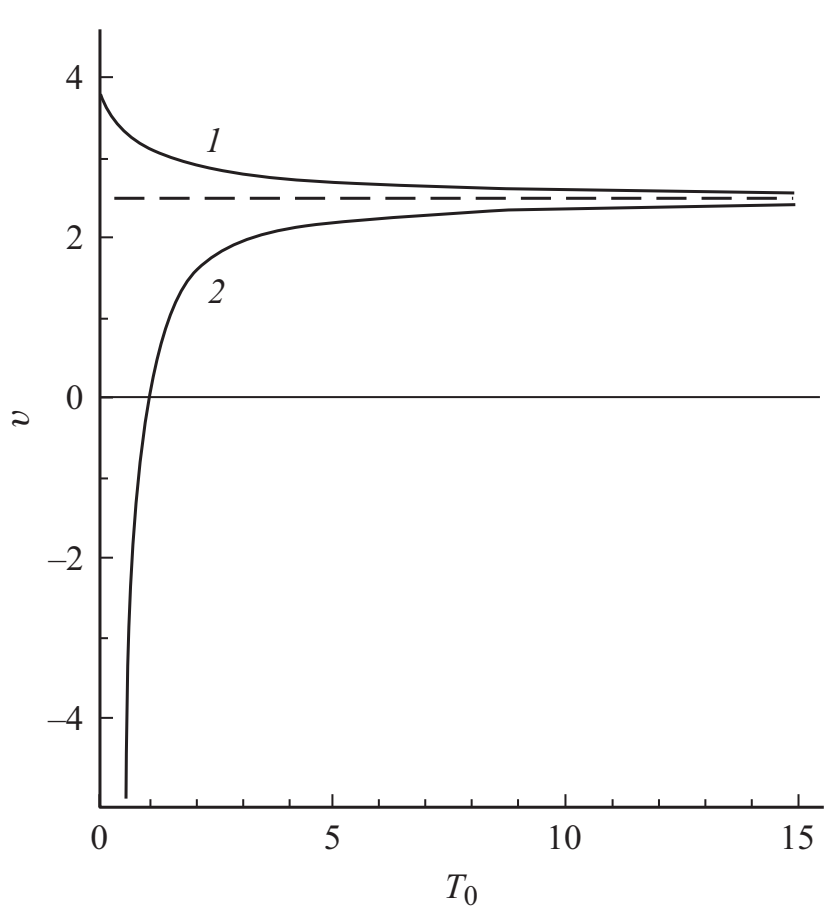

Рис. 3. Зависимости $v\left(T_{0}\right)$ при $d_{j}<0(1)$ и $d_{j}>0(2)$.

$\chi \rightarrow+\infty)$. Решение представляет собой ударную волну аналогичную стационарной ударной волне уравнения КдВ-Бюргерса [18].

Присутствие в уравнении (16) дополнительного нелинейного слагаемого не приводит к появлению принципиально новых решений. Нелинейные эффекты уравновешиваются диссипативными эффектами и дисперсией в рассматриваемой среде. Наличие дополнительной нелинейности не дает качественных изменений.

Зависимости скорости стационарной волны от начальной температуры изображены на рис. 3 для сред с дефектами типов „вакансия“ 1 и „межузлие“ 2 .

Для сред с вакансиями стационарная волна, распространяющаяся с найденной фиксированной скоростью (19), существует при любых положительных температурах. Для сред с межузлиями стационарная волна существует только при $T_{0}>T_{0}^{*}$, где $T_{0}^{*}-$ значение температуры, при котором скорость волны равна нулю.

Увеличение температуры $T_{0}$ приводит к увеличению скорости стационарной волны, если дефектами являются межузлия, и уменьшению, если дефектами являются вакансии. В среде с вакансиями волна распространяется быстрее, чем в среде с межузлиями. При больших температурах волны в обеих средах распространяются с почти одинаковыми скоростями

$$
v=\frac{1}{2 \varepsilon\left(1+\frac{\gamma \beta_{j}}{q_{\varepsilon} p_{j}}\right)} .
$$

\section{Частные случаи эволюционного уравнения}

Если в уравнении (15) диссипативное слагаемое мало по сравнению со всеми остальными слагаемыми уравнения (случай 1), то им можно пренебречь, и получим уравнение Кортевега-де Вриза с дополнительным нелинейным слагаемым

$$
\frac{\partial W}{\partial \eta}+\frac{a_{2}\left(1-a_{1}\right)}{2 \varepsilon} \frac{\partial^{3} W}{\partial \xi^{3}}+\frac{a_{3}}{2 \varepsilon}\left(W \frac{\partial W}{\partial \xi}-\frac{\partial}{\partial \xi}\left(W \frac{\partial W}{\partial \xi}\right)\right)=0 .
$$

Уравнение (21) имеет аналитическое решение в виде стационарной волны

$$
W(\chi)=\frac{a_{2}}{a_{3}}\left(1-a_{1}\right)\left(1-\operatorname{th}\left(\frac{\chi}{2}\right)\right),
$$

где $\chi$, как и прежде, бегущая координата, $\chi=\xi-v \eta, v-$ скорость стационарной волны, равная $v=a_{2}\left(1-a_{1}\right) / 2 \varepsilon$. Решение (22) найдено тем же методом, что и ранее полученное решение (20). Профиль волны имеет вид, аналогичный изображенному на рис. 1 - плавного скачка (кинк) между двумя значениями функции продольной деформации $W(\chi)$. Кинк имеет монотонное убывание и симметрию относительно точки перегиба.

Исследуя состояния равновесия $W_{0}=0, W_{0}=2 a_{2}(1-$ $\left.-a_{1}\right) / a_{3}$ на устойчивость, получаем, что в одном случае характеристическое уравнение имеет действительные корни разных знаков, что соответствует состоянию равновесия „седло“, в другом случае решением характеристического уравнения являются действительные равные положительные корни, что соответствует состоянию равновесия „неустойчивый вырожденный узел“. Решением уравнения (21) является стационарная ударная волна.

Известно, что решением уравнения Кортевега-де Вриза является стационарная волна типа „солитон“'. Движение на фазовой плоскости происходит из „седла“ в „седло“. В этом случае стационарная волна является результатом взаимодействия двух механизмов: нелинейности и дисперсии. Нелинейность дает укручение профиля волны с последующим опрокидыванием (волна Римана), а дисперсия дает расплывание профиля, поскольку разные гармоники волны (частоты) распространяются с разными скоростями.

Решением уравнения (21) является стационарная ударная волна, которая является результатом взаимодействия эффектов нелинейности и диссипации, несмотря на то, что уравнение содержит только дисперсионное и нелинейные слагаемые, а диссипативное слагаемое в уравнении отсутствует.

Из линеаризованного уравнения относительно малых возмущений $\left(W(\chi)=W_{0}+\tilde{W}(\chi)\right)$

$$
\frac{\partial \tilde{W}}{\partial \eta}+\frac{a_{2}\left(1-a_{1}\right)}{2 \varepsilon} \frac{\partial^{3} \tilde{W}}{\partial \xi^{3}}+\frac{a_{3} W_{0}}{2 \varepsilon}\left(\frac{\partial \tilde{W}}{\partial \xi}-\frac{\partial^{2} \tilde{W}}{\partial \xi^{2}}\right)=0
$$


видно, что диссипация в уравнении (21) присутствует неявно. Последнее слагаемое в уравнении (23) является диссипативным.

Таким образом, дополнительное слагаемое в (21) является диссипативно-нелинейным. Наличие этого слагаемого в уравнении и делает возможным существование решений в виде стационарных ударных волн.

Если в уравнении (15) дисперсионное слагаемое мало по сравнению со всеми остальными слагаемыми (случай 2), то получаем уравнение Бюргерса с дополнительным нелинейным слагаемым

$\frac{\partial W}{\partial \eta}+\frac{\left(1-a_{1}\right)}{2 \varepsilon} \frac{\partial^{2} W}{\partial \xi^{2}}+\frac{a_{3}}{2 \varepsilon}\left(W \frac{\partial W}{\partial \xi}-\frac{\partial}{\partial \xi}\left(W \frac{\partial W}{\partial \xi}\right)\right)=0$.

Это возможно, когда $a_{2} \rightarrow 0$, т.е. диффузия очень мала $\left(D_{j} \rightarrow 0\right)$.

Уравнение (24) можно отнести к обобщенным невозмущенным уравнениям Бюргерса. В отличие от уравнения Бюргерса [18], здесь присутствует еще один тип нелинейности, причем обе нелинейности проявляются в равной степени. Уравнение (24) имеет стационарное решение в виде ударной волны. Учитывая граничные условия (функция на бесконечностях имеет разные значения)

$$
W(\chi)=\left\{\begin{array}{ll}
W_{1}, & \chi \rightarrow+\infty \\
W_{2}, & \chi \rightarrow-\infty
\end{array}\left(W_{2}>W_{1}\right),\right.
$$

находим решение уравнения стационарных волн

$$
\begin{aligned}
\chi= & \frac{2\left(1-a_{1}-a_{3} W_{1}\right)}{a_{3}\left(W_{2}-W_{1}\right)} \ln \left(W-W_{1}\right)-\frac{2\left(1-a_{1}-a_{3} W_{2}\right)}{a_{3}\left(W_{2}-W_{1}\right)} \\
& \times \ln \left(W_{2}-W\right),
\end{aligned}
$$

где $W=W(\chi), \chi-$ бегущая координата, $\chi=\xi-v \eta$, скорость нелинейной волны определяется выражением $v=a_{3}\left(W_{1}+W_{2}\right) / 4 \varepsilon$. Профиль стационарной ударной волны $W(\chi)$ и график производной изображены на рис. 4. График производной отображен в верхнюю полуплоскость и смещен по оси ординат на $W_{1}$.

Параметры ударной волны, возникающей в результате взаимной компенсации эффектов нелинейности и диссипации, связаны соотношением

$$
\frac{a_{3} A}{1-a_{1}}\left(\frac{\Delta}{2}-1\right)=\text { const }
$$

где $A=W_{2}-W_{1}-$ амплитуда ударной волны, $\Delta-$ характерная ширина фронта ударной волны. Скорость нелинейной волны зависит от амплитуды и ширины фронта волны.

Рассмотрим случай, когда в уравнении (15) параметр $a_{1}=1$ (случай 3). Это возможно, если дефектами являются межузлия $\left(d_{i}>0\right)$. Для вакансий $0<a_{1}<1$. Для межузлий $a_{1}>0$, причем, если влияние температур мало, то $a_{1} \gg 1$.

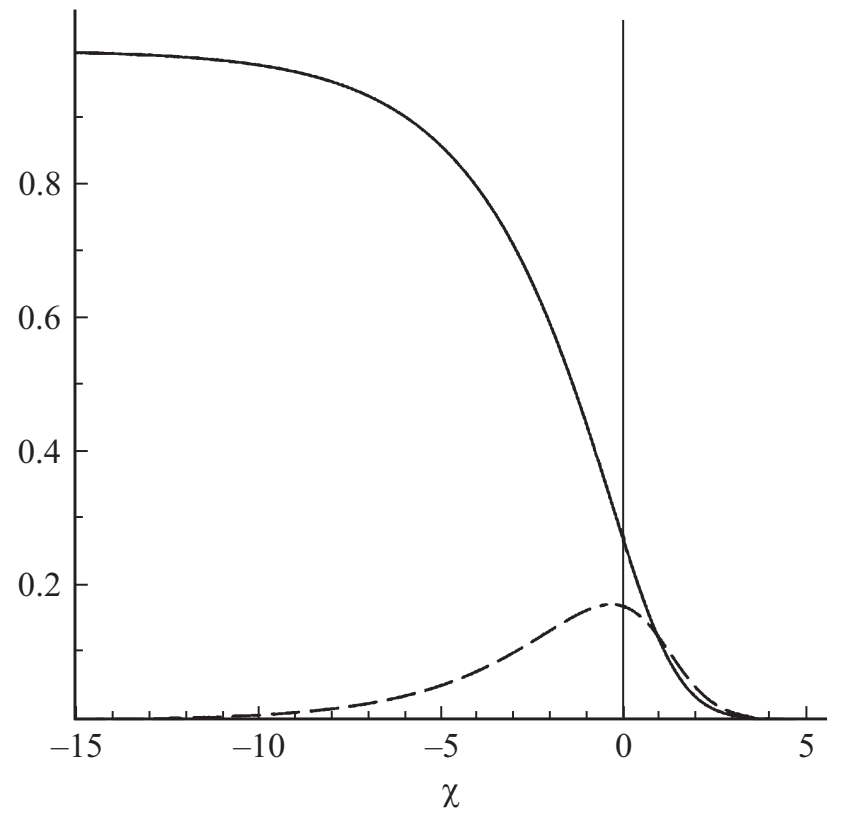

Рис. 4. Зависимости $W(\chi)$ (сплошная линия), $W^{\prime}(\chi)$ (штриховая линия).

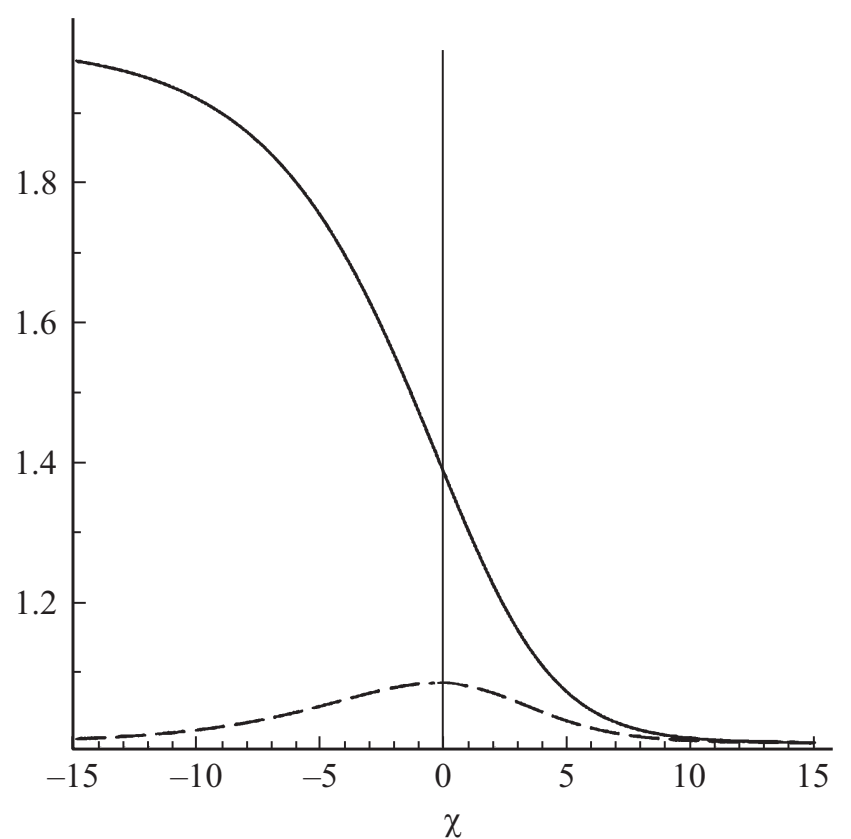

Рис. 5. Зависимости $W(\chi)$ (сплошная линия), $W^{\prime}(\chi)$ (штриховая линия).

Если $a_{1}=1$, то получаем уравнение, в котором отсутствуют диссипативное и дисперсионное слагаемые, но сохраняются нелинейные слагаемые

$$
\frac{\partial W}{\partial \eta}+\frac{a_{3}}{2 \varepsilon}\left(W \frac{\partial W}{\partial \xi}-\frac{\partial}{\partial \xi}\left(W \frac{\partial W}{\partial \xi}\right)\right)=0
$$

Уравнение (25) имеет дополнительное нелинейное слагаемое относительно уравнения Римана. Нелинейности 
в уравнение входят с одинаковыми коэффициентами и разными знаками.

Уравнение (25) имеет решение

$$
\chi=\frac{2}{\left(W_{2}-W_{1}\right)}\left[W_{2} \ln \left(W_{2}-W\right)-W_{1} \ln \left(W-W_{1}\right)\right]
$$

в виде стационарной ударной волны, распространяющейся со скоростью $v=\left(a_{3} / 4 \varepsilon\right)\left(W_{1}+W_{2}\right)$ вдоль координаты $\chi=\xi-v \eta$. Профиль волны изображен на рис. 5.

Ширина фронта ударной волны является постоянной величиной $(\Delta=$ const $)$, т.е. не зависит от амплитуды волны и исходных параметров системы. В этом случае к появлению ударной волны приводит взаимодействие разнородных нелинейностей. Линеаризация уравнения (25) относительно малых возмущений

$$
\frac{\partial \tilde{W}}{\partial \theta}+b_{3}\left(\frac{\partial \tilde{W}}{\partial \xi}-\frac{\partial^{2} \tilde{W}}{\partial \xi^{2}}\right) W_{0}=0
$$

показывает, что второе нелинейное слагаемое привносит затухание. Это затухание способствует появлению стационарной ударной волны в системе. Уравнение (25) обладает свойством уравнения Бюргерса, несмотря на то, что явно диссипативные слагаемые в этом уравнении отсутствуют.

\section{Заключение}

На основе анализа точного аналитического решения эволюционного уравнения относительно смещений частиц среды показано, что вакансии и межузлия способствуют формированию стационарных ударных волн в среде. Показано, что ударные волны могут быть симметричного и несимметричного профиля.

\section{Финансирование работы}

Работа выполнена при финансовой поддержке гранта Правительства Российской Федерации (договор № 14.Y26.31.0031).

\section{Конфликт интересов}

Авторы заявляют, что у них нет конфликта интересов.

\section{Список литературы}

[1] Мирзоев Ф.Х., Панченко В.Я., Шелепин Л.А. // УФН. 1996. T. 166. № 1. C. 3-32. DOI: 10.3367/UFNr.0166.199601a.0003 [Mirzoev F.Kh., Panchenko V.Ya., Shelepin L.A. // Phys. Usp. 1996. Vol. 39. N 1. P. 1-29. DOI: 10.1070/PU1996v039n01ABEH000125]

[2] Бурлак Г.Н., Островский И.В. // Письма в ЖТФ. 1997. T. 23. Вып. 18. C. 69-74 [Burlak G.N., Ostrovskii I.V. // Tech. Phys. Lett. 1997. Vol. 23. N 9. P. 725-726. DOI: https://doi.org/10.1134/1.1261669]
[3] Ерофеев В.И., Ромамов В.П. // Письма в ЖТФ. 2002. T. 28. Вып. 6. С. 6-11. [Erofeev V.I., Romashov V.P. // Tech. Phys. Lett. 2002. Vol. 28. N 3. P. 218-220. DOI: https://doi.org/10.1134/1.1467280]

[4] Ерофеев В.И. // Письма в ЖТФ. 2008. Т. 34. Вып. 4. С. 32 36. [Erofeev V.I. // Tech. Phys. Lett. 2008. Vol. 34. N 2. P. 150 152. DOI: https://doi.org/10.1134/S1063785008020193]

[5] Шекоян А.В. // Письма в ЖТФ. 2009. Т. 35. № 7. С. 93-97. [Shekoyan A.V. // Tech. Phys. Lett. 2009. Vol. 35. N 4. P. 337339. DOI: https://doi.org/10.1134/S1063785009040142]

[6] Багдоев А.Г., Ерофеев В.И., Шекоян А.В. Линейные и нелинейные волны в диспергирующих сплошных средах. М.: Физматлит, 2009. 320 с. [Bagdoev A., Erofeyev V., Shekoyan A. Wave Dynamics of Generalized Continua. Spinger: Berlin, Heidelberg, 2016. 274 p.

DOI: https//doi.org/10.1007/978-3-642-37267-4]

[7] Ерофеев В.И., Кажаев В.В. // ЖТФ. 2010. Т. 80. Вып. 4. C. 149-151. [Erofeev V.I., Kazhaev V.V. // Tech. Phys. 2010. Vol. 55. N 4. P. $580-583$.

DOI: https://doi.org/10.1134/S1063784210040262]

[8] Сарафанов Г.Ф. Коллективные и волновые эффекты в ансамбле дислокаций при пластической деформации металлов. Н. Новгород: Изд-во Литера, 2010. 359 с.

[9] Ерофеев В.И., Мальханов А.О. // Физическая мезомеханика. 2017. Т. 20. № 4. С. 69-76. [Erofeev V.I., Malkhanov A.O. // Phys. Mesomech. 2019. Vol. 22. N 3. P. $173-180$ DOI: https://doi.org/10.1134/S1029959919030019]

[10] Мирзоев Ф.Х., Шелепин Л.А. // ЖТФ. 2001. Т. 71. Вып. 8. C. 23-26 [Mirzoev F., Shelepin L.A. // Tech. Phys. 2001. Vol. 46. N 8. P. 952-955.

DOI: https://doi.org/10.1134/1.1395114]

[11] Ерофеев В.И., Артамонова О.А. // Труды ХХІІ Сессии Российского акустического общества и Научного совета по акустике РАН. М.: ГЕОС, 2010. Т. 1. С. 159-159.

[12] Мирзаде Ф.Х., Шелепин Л.А. // Акустический журнал. 2007. Т. 53. № 6. С. 766-773. [Mirzade F.Kh., Shelepin L.A. // Acoustical Phys. 2007. Vol. 53. N 6. P. 676682. DOI: https://doi.org/10.1134/1.1395114]

[13] Erofeev V.I., Leontieva A.V., Malkhanov A.O. // IOP Conference Series: Materials Science and Engineering. 2017. Vol. 208. N 012017. P. 1-8.

[14] Ерофеев В.И., Леонтьева А.В., Мальханов А.О. // Известия РАН. Серия физическая. 2018. Т. 82. № 5. С. 591-596. [Erofeev V.I., Leonteva A.V., Malhanov A.O. // Bulletin of the Russian Academy of Sciences: Phys. 2018. Vol. 82. N 5. P. 520-525. DOI: https://doi.org/10.3103/S1062873818050088]

[15] Порубов А.В. Локализация нелинейных волн деформации. М.: Физматлит, 2009. 208 с.

[16] Кудрямов Н.А. Аналитическая теория нелинейных дифференциальных уравнений. Москва-Ижевск: Институт компьютерных исследований, 2004. 360 с.

[17] Кудряшов Н.A. Методы нелинейной математической физики. Долгопрудный: Интеллект, 2010. 368 с.

[18] Кадомиев Б.Б., Карпман В.И. // УФН. 1971. Т. 103. № 2. С. 193-232. DOI: 10.3367/UFNr.0103.197102a.0193 [Kadomtsev B.B., Karpman V.I. // Sov. Phys. Usp. 1971. Vol. 14. N 1. P. 40-60. DOI: 10.1070/PU1971v01ABEH004441] 